\title{
Editorial
}

\section{Politik in Deutschland}

Die bundesdeutsche Politik der letzten fünf Jahre bietet eine seltsame Ironie. Wurde in den 80er Jahren das Projekt eines sozialen und ökologischen Umbaus der Industriegesellschaft allein schon aus finanziellen Gründen von den Regierenden als illusionär abgetan, so mobilisiert dieselbe Regierung für die Übertragung der kapitalistischen Marktordnung auf Ostdeutschland heutzutage Beträge, von denen die linken und grünen Reformpolitiker damals nicht einmal zu träumen wagten. Nach fünf Jahren desaströser Vereinigungspolitik mag man sich darüber streiten, wessen Vorstellungen illusionärer waren.

Die Bonner Visionäre richteten ihren Blick zunächst auf eine verklärte westdeutsche Nachkriegsgeschichte. Nach dem Modell von Währungsreform und Wirtschaftswunder wollte man auch im Osten die Marktkräfte »entfesseln«. Die politischen Entscheidungsträger hofften, sich nach Durchsetzung der richtigen Grundentscheidungen auf einen »ordnungspolitischen Rahmen« zurückziehen zu können. Die Sanierung der Volkswirtschaft und die Angleichung der Lebensverhältnisse sollte sich durch Veräußerung des ehemals »volkseigenen« Vermögens selbst finanzieren. Der zunächst überwie- gend kreditfinanzierte $"$ Fonds Deutsche Einheit « und die einheitsbedingt steigende Staatsverschuldung sollten sich durch erwartete Steuermehreinnahmen innerhalb weniger Jahre konsolidieren. Das Ausmaß der Verschuldung und die zukünftig anfallenden Kosten wurden freilich vorsichtshalber hinter einer Vielzahl kaum noch durchschaubarer Sonderhaushalte versteckt - was bei einer Aktiengesellschaft den Verdacht des Bilanzbetrugs nahegelegt hätte.

Nachdem die dem Markt zugesprochenen »selbstorganisierenden « $\mathrm{Po}$ tenzen ausblieben, nachdem der Umbau der ostdeutschen Industrie sich nicht »selbst finanzierte«, ging die Bonner Politik dazu über, in einem bislang nicht gekannten Umfang Ressourcen zu mobilisieren und umzuverteilen - blieb allerdings in einem ideologischen Bezugsrahmen gefangen, der eine langfristig angelegte Industrie- und Infrastrukturpolitik nicht zuläßt. Das Resultat war ein gedankenloses, prozyklisch wirkendes Flickwerk, zusammengestückt mit Blick auf die nächsten Wahlen. Auf der Höhe des Vereinigungsbooms wurde die Nachfrage durch Steuernachlässe und konsumptive Transfers stimuliert; die beginnende Rezession wurde durch Zinssteigerungen und eine erhöhte 
Abgabenlast zur ersten gesamtdeutschen Krise verschärft. Die Kumulation wirtschaftspolitischer Fehlentscheidungen läßt mehr als fraglich erscheinen, ob es einen Ausweg aus dem vicious circle gibt, den Kurt Hübner in diesem Heft für die fünf neuen Länder diagnostiziert: ein "zweites deutsches Wirtschaftswunder« ist östlich wie westlich der Elbe außer Sicht.

Dieser Abwärtszirkel war demnach nicht allein den vielbeschworenen »40 Jahren Sozialismus« geschuldet. Die konservative Vereinigungspolitik hat maßgeblich an ihm mitgestrickt. Wenn der Bonner Aktionismus ein Muster aufwies, so bestand es darin, die Folgelasten der einsamen Entscheidungen des Kanzleramts auf Institutionen abzuwälzen, die für den Umbau einer Gesellschaftsordnung denkbar ungeeignet sind. De facto wurde Wirtschaftspolitik in Ostdeutschland zum guten Teil von der Treuhandanstalt improvisiert, einer Institution, deren vorrangiges Ziel eine Privatisierung nach betriebwirtschaftlichen Gesichtspunkten war. Der Umgang mit den inflationären Folgen der Währungsumstellung, die auf eine Entwertung der Betriebsschulden verzichtete, wurden der Geldmengensteuerung der Bundesbank überlassen. Die aufgrund des politischen Umtauschsatzes sprunghaft gestiegenen ostdeutschen Lohnkosten sollten durch die Zurückhaltung der Gewerkschaften kompensiert werden. Die enormen sozialpolitischen
Anpassungskosten wurden der Arbeitslosen- und Rentenversicherung sowie den Gemeindekassen aufgebürdet. Die Diskussion um eine gesamtdeutsche Verfassung fand nicht in einer breiten Öffentlichkeit, sondern als Expertenanhörung vor einer fragwürdig legitimierten Kommission statt (vgl. dazu in diesem Heft den Beitrag von Uwe Berlit).

Die Bonner Externalisierung von Politik auf Bundesbank, Tarifordnung, Sozialsysteme und Kommunen, d.h. auf das westdeutsche Institutionengefüge, das dem Osten im Prozeß einer konservativen Modernisierung übergestülpt wurde, provozierte einen fatalen Effekt: eine rückwirkende Dynamik, welche die für den relativen Erfolg des westdeutschen Modells verantwortlichen Kompromißstrukturen durch Überbeanspruchung zunehmend erodiert. Unter Hinweis auf den ostdeutschen Ausnahmezustand wird versucht, die sozialen Netze immer dünner zu knüpfen. Die Sozialversicherungsfonds werden zur Finanzierung des nationalen Projekts herangezogen, was zu Beitragserhöhungen und Leistungsminderung führt und die soziale Verankerung der Sicherungssysteme schädigt. Von einer ähnlichen Delegitimierung ist auch ein zentrales Prinzip der industriellen Beziehungen bedroht: die Tarifautonomie. Das ostdeutsche Niveau der Lohnstückkosten, der Hauptseire nach Folge der Währungsunion, wurde zu hohen Tarifabschlüssen angelastet. Die daraufhin von der 
Regierung unterstützten Rufe nach einer Öffnung von Tarifklauseln laufen auf einen Eingriff in die Tarifautonomie hinaus. Die Gewerkschaften sind darüber in eine schwierige Position geraten: sie müssen das politische Versprechen einer sozialen Angleichung als Tarifforderung deklarieren. Bis jetzt jedenfalls ist es der Regierung in erstaunlichem Ausmaß gelungen, die Verantwortung für die wirtschaftliche Misere den Gewerkschaften zuzuschieben - obwohl sie nichts anderes praktisch einzulösen versuchen, als die im letzten Wahlkampf versprochene Angleichung der Löhne.

Die deutsche Regierung ist zur Gefangenen ihres eigenen Dogmas geworden. Mangels eines expliziten, auf breiter Teilnahme beruhenden politischen Rahmens kann sie den Umbau der ostdeutschen Wirtschaft nur noch als »nationale Aufgabe « formulieren und mit der für eine moderne Gesellschaft eigentümlichen Gesinnungszumutung an ihr Publikum unterstreichen, doch endlich die »innere Einheit « zu wollen. Die politische Kontroverse über sinnvolle verkehrs-, bildungs-, energie- und arbeitsmarktpolitische Zielsetzungen wurde in die nationalistische Semantik von »Schicksal«, "historischen Augenblicken « und (erfahrungsgemäß höchst ungleich verteilten) »notwendigen Opfern« aufgelöst.

Außenpolitisch kommt der neudeutsche Nationalismus freilich nicht in den alten Kleidern daher, sondern gibt sich als diplomatischer Sachverstand. Deutschland solle endlich zu seiner »Verantwortung « für die Welt stehen. Dieser Sachverstand aber läßt bereits für Europa nichts Gutes ahnen. Schon mit der deutschdeutschen Währungsunion wurde ein Sonderweg eingeschlagen, der mit einer europäischen Zentralbank kaum mehr gangbar gewesen wäre. So aber wurde das EWS als monetärer Rahmen einer gemeinsamen europäischen Wirtschaftspolitik gesprengt. Um die Inflationsgefahren der Einheitspolitik zu begrenzen, wurden die deutschen Zinsen auf ein Niveau getrieben, das den Nachbarländern nur die Wahl ließ, entweder durch ein vergleichbar hohes Zinsniveau die Rezessionstendenzen zu verstärken oder dem Kurssturz der eigenen Währung zuzusehen. Die erste außenpolitische Großtat des neuen Deutschland, die forcierte Anerkennung Sloweniens und Kroatiens, heizte einen ohnehin schon gefährlichen Konflikt weiter an und unterlief von vornherein eine gemeinsame EG-Außenpolitik.

Daß die »internationale Verantwortung « nicht zuletzt militärisch gemeint ist, zeigt der Somalia-Einsatz der Bundeswehr. Die zuweilen operettenhaft anmutenden Begleitumstände (die deutschen Einheiten sollten die Versorgung nie eingetroffener indischer Verbände sicherstellen und ihrerseits von italienischen Einheiten gesichert werden etc.) sollten nicht darüber hinweg- 
täuschen, daß mit diesem ersten »out of area« Einsatz der Bundeswehr ein Tabu der deutschen Nachkriegspolitik durchbrochen ist. Die Außenpolitik ist zum Experimentierfeld dafür geworden, was verfassungsmäßig so alles drin ist - oder wo es nachträglicher Änderungen bedarf. Beim nächsten Golfkrieg dürfen vielleicht auch deutsche Landser mitschießen. Allerdings kann man vom inzwischen zweitgrößten Waffenexporteur wohl auch verlangen, daß er die Qualität seines Geräts durch eigenen Einsatz unter Beweis stellt.

Nach innen artikuliert sich die Bonner Politik auf spezifisch deutsche Weise - mit dem wirtschaftsnationalistischen Appell, den »Standort《 zu wahren. Der korporative Rahmen der sozialen Marktwirtschaft soll das zustande bringen, was die Deregulierungen des vergangenen Jahrzehnts nicht vermochten: die allgemeinen Lohn- und Lohnnebenkosten sollen jetzt mit Zustimmung der Gewerkschaften gesenkt werden.

In den Hintergrund wird gedrängt, $\mathrm{daß}$ die Vereinigungspolitik selbst zum größten »Standortrisiko« geworden ist. Mit der Finanzierung der Vereinigungslasten über die Sozialkassen hat niemand anders als der Staat die Lohnnebenkosten um mehrere Prozentpunkte hochgetrieben. Die Inflation geht weit überwiegend auf die Anhebung administrierter Preise, d.h. auf Gebührenund Steuererhöhungen, sowie auf in die frühen 80 er Jahren zurückrei- chende wohnungsbaupolitische Versäumnisse zurück, die die gegenwärtigen Mietsteigerungen erst ermöglichen. Für die im internationalen Vergleich gestiegenen Stückkosten ist in erster Linie der aufgrund des Zinsniveaus gestiegene Außenwert der Währung verantwortlich.

Wie so oft, wird auch in der gegenwärtigen Krise der nationale Appell als Ersatz für gescheiterte Politik eingesetzt, sei er an die Opferbereitschaft der Bürger, sei er an das Pflichtbewußtsein der Gewerkschaften adressiert. Die Politik läßt sich auf die riskante Unterscheidung zwischen dem $\gg$ gesunden Nationalismus « der Bürger, die den Fernsehabend mit Hymne und wehender Flagge beschließen, und der gewaltsamen »Identitäts«-Behauptung jener ein, die gegen die »Überfremdung« der »deutschen Kultur« handgreiflich vorgehen. In der verkehrten Welt der bundesdeutschen Politik gilt auch dieser Rassismus noch als Anlaß, die » ̈̈ngste« der rechtsradikalen Klientel vor ausländischer Konkurrenz um Wohnung und Arbeitsplatz »ernst zu nehmen«. Diese Ängste beruhen, wie Andrea Fischer in ihrem Beitrag zeigt, auf Fiktionen. Gleichwohl scheinen sie geeignet, die fällige Kritik an sozialund wohnungsbaupolitischen Versäumnissen und das eigene Versagen vor den gestiegenen Anforderungen des Arbeitsmarkt auf anderssprachige Mitkonkurrenten umzuleiten - und die Regierung von einer Integrationspolitik zu entlasten. 
Daß dieses Interpretationsangebot in der ethnisch definierten Staatszugehörigkeit des Grundgesetzes angelegt ist, sollte den $\gg$ Verfassungspatriotismus « kritischer Intellektueller in Grenzen halten. Wie gerade die Asylrechtspraxis zeigt, operiert das Recht nicht »selbstbezüglich geschlossen«, sondern führt gegebenenfalls massive Ausgrenzungen herbei. Wie wenig die gewaltsame und ausgrenzende Seite des Rechts in der avancierten rechtssoziologischen Diskussion thematisiert wird auch das gehört zum notorischen Idealismus des deutschen Bewußtseins - stellt Wolf-Dieter Narr in seinem Review-Essay zu den jüngsten Publikationen von Jürgen Habermas und Niklas Luhmann dar. $\mathrm{Zu}$ den weniger rechtlich als sozial ausgegrenzten Verlierern der Einheit gehören die Frauen. Wie es möglich ist, dieser Tatsache ins Auge zu sehen, ohne zugleich den »ewigen « weiblichen Opferstatus zu beklagen, zeigt der Beitrag von Brigitte Young.

Der praktische Verlauf der deutschen Vereinigung hat zwei Axiome der gängigen Transformationökonomie in Frage gestellt: Märkte entfalten sich alles andere als »selbstorganisiert«; die Privatisierung des ehemaligen staatssozialistischen Eigentums ist keineswegs der natürliche Weg zu einem wirtschaftlichen Aufschwung. Mit den Aufsätzen von Frank Hahn und David Stark wird die in PROKLA 89 begonnene Auseinandersetzung um die theoreti- schen Grundlagen der Transformationsökonomie fortgesetzt. Die von Hahn gestellte Frage nach der Relevanz der »Allgemeinen Gleichgewichtstheorie« für den Übergang von Plan- zu Marktwirtschaften ist insofern von Belang, als die neoklassischen Politikempfehlungen von Jeffrey Sachs bis zum Internationalen Währungsfonds ihre wissenschaftliche Legitimation aus diesem Paradigma beziehen. Hahn, selbsi einer der prominentesten Vertreter dieses Ansatzes, zeigt, daß sogar unter den Prämissen der Allgemeinen Gleichgewichtstheorie ein marktgläubiger Verzicht auf die sichtbare Hand der Politik die Übergangskosten nur erhöht. David Starks Beitrag zeigt darüber hinaus wie illusorisch die klar geschnittene Alternative zwischen privatem und öffentlichem Sektor ist: im Prozeß der »Vermarktwirtschaftlichung « überkreuzen sich alte und neue Eigentumstitel zu einer neuen Eigentumsform, die Stark »rekombiniertes Eigentum « nennt. Daß es um die theoretischen Fundamente der nach wie vor nicht nur akademisch, sondern auch politisch einflußreichen neoklassischen Theorie nicht allzu gut bestellt ist, zeigt der Artikel von Klaus Schabacker. Er rekonstruiert Sraffas Fundamentalkritik an der Neoklassik und skizziert mögliche Verbindungen zwischen Sraffas Ansatz und Positionen von Marx und Keynes. 